\title{
A Generalization of Partition Identities for First Differences of Partitions of $n$ Into at Most $m$ Parts
}

\author{
Acadia Larsen \\ Department of Mathematics \\ University of California, Davis \\ Davis, California, U.S.A. \\ alarsen@math.ucdavis.edu
}

Submitted: Sep 22, 2018; Accepted: Aug 18, 2020; Published: Jul 16, 2021

(C) The author. Released under the CC BY license (International 4.0).

\begin{abstract}
We show for a prime power number of parts $m$ that the first differences of partitions into at most $m$ parts can be expressed as a non-negative linear combination of partitions into at most $m-1$ parts. To show this relationship, we combine a quasipolynomial construction of $p(n, m)$ with a new partition identity for a finite number of parts. We prove these results by providing combinatorial interpretations of the quasipolynomial of $p(n, m)$ and the new partition identity. We extend these results by establishing conditions for when partitions of $n$ with parts coming from a finite set $A$ can be expressed as a non-negative linear combination of partitions with parts coming from a finite set $B$.
\end{abstract}

Mathematics Subject Classifications: 11P84, 05A17, 05A19

\section{Introduction}

A partition $\lambda$, of a non-negative integer $n$ is a non-increasing sequence of parts $\lambda_{1}, \ldots, \lambda_{k}$ such that the parts sum to $n$. We denote this $\lambda \vdash n$ and is read " $\lambda$ is a partition of $n$ ". Let $p(n)$ be the function which enumerates all partitions of $n$. The first example of partitions traces back to Leibniz in a letter to Bernoulli. While neither did much work on the subject, Euler took up the subject giving the first deep results into the theory of partitions, such as the following relationship for partitions of $n$ into at most $m$ parts, $p(n, m)$.

Proposition 1 (Euler). For all non-negative integer $n$ and all positive integers $m$,

$$
p(n, m)=p(n, m-1)+p(n-m, m) .
$$

The proof follows from generating function for $p(n, m)$ which is

$$
\sum_{n=0}^{\infty} p(n, m) q^{n}=\prod_{i=1}^{m} \frac{1}{1-q^{i}}=\frac{1}{(q ; q)_{m}}
$$


where $(q ; q)_{m}$ is the $q$-pochhammer symbol defined as

$$
(a ; x)_{n}=\prod_{i=0}^{n-1}\left(1-a x^{i}\right) .
$$

Partition identities, expressing one kind of partitions as another, have been extensively studied by mathematicians since Euler. In this paper, we examine first differences of partitions $p(n, m)-p(n-1, m)$ for prime power $m$. We capture several known partition identities found in [1] and [6] as well as previously unknown partition identities.

Example 2. For $k \geqslant 0$,

$$
\begin{aligned}
p(6 k+3,3)-p(6 k+2,3) & =p(2 k+1,2) \\
p(12 k+5,4)-p(12 k+4,4) & =p(6 k+1,3) \\
p(60 k+0,5)-p(60 k-1,5) & =p(12 k+0,4)+p(12 k-1,4)+4 p(12 k-2,4) \\
& +5 p(12 k-3,4)+7 p(12 k-4,4)+4 p(12 k-5,4) \\
& +3 p(12 k-6,4) .
\end{aligned}
$$

Lines (4) and (5)are known ([1], [6]) while line (6) was previously unknown. These identities are special cases of the following theorem.

Theorem 3. Let $s$ be a prime. If $m=s^{x}$ where $x$ is a positive integer and for $k \geqslant 0$, $0 \leqslant j<l \mathrm{~cm}(\mathrm{~m})$, then

$$
p(l c m(m) k+j, m)-p(l c m(m) k+j-1, m)=\sum_{i \geqslant 0} c_{r+s i} p\left(l c m(m-1) k+l^{\prime}-i, m-1\right)
$$

where $l^{\prime}$ and $r$ satisfy $j=l^{\prime} s+r$ with $0 \leqslant r<s, l c m(m)$ is the least common multiple of the numbers 1 through $m$, and $c_{r+i s}$ are the coefficients of some polynomial $G(q)$.

$G(q)$ is given in Section 3 in the statement of Lemma 13. Identities similar to Theorem 3 have been explored [3], [2], and [5].

Let $A$ be a finite set of positive integers not necessarily distinct. We denote the number of partitions of $n$ with parts from $A$ as $p(n, A)$. Its generating function is given by

$$
\sum_{n=0}^{\infty} p(n, A) q^{n}=\prod_{i \in A} \frac{1}{1-q^{i}} .
$$

We provide the following combinatorial interpretation for a partition from a finite set $A$. Let $\alpha_{i}$ denote the number of copies of an integer $i \in A$. For a partition $\lambda$ into parts from $A$, a part of size $i$ can have colors $1,2, \ldots, \alpha_{i}$ and we use the notation

$$
\begin{aligned}
\lambda & =\left(a_{i_{1}, 1}, a_{i_{1}, 2}, \ldots, a_{i_{1}, \alpha_{i_{1}}}, \ldots, a_{i_{k}, 1}, a_{i_{k}, 2}, \ldots, a_{i_{k}, \alpha_{i_{k}}}\right) \\
& =\left(a_{1,1}, a_{1,2}, \ldots, a_{1, \alpha_{1}}, \ldots, a_{k, 1}, \ldots a_{k, \alpha_{k}}\right)
\end{aligned}
$$


where $i_{1}, i_{2}, \ldots, i_{k}$ are the distinct elements of $A$ in order of least to greatest and $a_{t, s}$ is the number of parts of size $i_{t}$ in color $s$. We remark that $p(n, m)=p(n,\{1,2, \ldots, m\})$ and it is assumed throughout that $m$ in place of $A$ is the set of positive integers 1 through $m$.

In this context, Theorem 3 asserts that when $m$ is a prime power and that if $A=$ $\{2,3, \ldots, m\}$ and $B=\{1,2, \ldots, m-1\}$ that $p(n, A)$ can be expressed as a non-negative linear combination of $p\left(n_{1}, B\right)$ for some finite collection of $n_{1}<n$. The techniques used to prove Theorem 3 can be applied to determine when partitions with parts from a finite set positive integers $A$ can be expressed as a non-negative linear combination of partitions with parts from a finite set positive integers $B$.

The paper is organized as follows: in Section 2, we establish a quasipolynomial for $p(n, A)$ and provide a proof that "counts" $p(n, m)$ similar to [7]. Next in Section 3, we build intuition for Theorem 3 by providing a new proof of the case $m=4$ that highlights useful generating function arithmetic. We prove several important lemmas and propositions regarding factorization of generating functions for particular sets of partitions. With that, we apply the results of Section 2 to prove Theorem 3. Lastly, in Section 4 introduce a group structure on a finite set of partitions and introduce conditions in which partitions with parts from a finite set positive integers $A$ can be expressed as a non-negative linear combination of partitions with parts from a finite set positive integers $B$.

Through the proof of Theorem 3, providing a quasipolynomial for $p(n, A)$, and Section 4 , we offer a combinatorial framework discussing $p(n, A)$. This framework is equipped with a group structure that arises naturally in the course of proving Theorem 3.

\section{Establishing a quasipolynomial for $p(n, A)$}

In this section, we summarize several arguments for creating quasipolynomial for $p(n, A)$.

Definition 4. A quasipolynomial is a piecewise integer function, $f$, such that there are polynomials $f_{0}(k), f_{1}(k), \ldots, f_{j-1}(k)$ with rational coefficients, called constituents, such that

$$
f(k)= \begin{cases}f_{0}(k) & \text { if } k \equiv 0 \quad(\bmod j) \\ f_{1}(k) & \text { if } k \equiv 1 \quad(\bmod j) \\ \vdots & \\ f_{j-1}(k) & \text { if } k \equiv j-1 \quad(\bmod j) .\end{cases}
$$

The number of constituents, $j$, of the quasipolynomial is called the period.

We refer the reader to [17] for further reading about quasipolynomials. The particular technique in Theorem 5 that we show has been presented a number of times, notably a integral aspect of the geometry of Ehrhart in [8], as a consequence of generating functions in [9] and [18], and more recently in [4], [5], [6], and [15]. Other methods for creating quasipolynomials of $p(n, m)$ rely on partial fraction decomposition of rational functions such as in [14] and [16].

We start by generalizing the notation of $\operatorname{lcm}(m)$ in the previous section. For a finite set of positive integers, $A$, we define $\operatorname{lcm}(A)$ to be the least common multiple of all the 
elements of $A$. For example $\operatorname{lcm}(3)=\operatorname{lcm}(\{1,2,3\})=6$. Let $d$ count the number of elements in $A$. We define the polynomial $E_{A}(q)$ by

$$
E_{A}(q)=\prod_{i \in A}\left(\sum_{j=0}^{\frac{\operatorname{lcm}(A)-i}{i}} q^{i j}\right)=\sum_{x=0}^{d \operatorname{lcm}(A)-\sum_{i \in A} i} a_{x} q^{x}
$$

Furthermore, $E_{A}(q)$ has the following property,

$$
\prod_{i \in A}\left(1-q^{i}\right) E_{A}(q)=\left(1-q^{\operatorname{lcm}(A)}\right)^{d} .
$$

Lastly, we remark that it is known that for $d$ a non-negative integer,

$$
\frac{1}{(1-q)^{d}}=\sum_{k=0}^{\infty}\left(\begin{array}{c}
k+d-1 \\
d-1
\end{array}\right) q^{k}
$$

Theorem 5. For all $k \geqslant 0$ and for $0 \leqslant j<\operatorname{lcm}(A)$,

$$
p(\operatorname{lcm}(A) k+j, A)=\sum_{t \geqslant 0} a_{j+l c m(A) t}\left(\begin{array}{c}
k-t+(d-1) \\
d-1
\end{array}\right) .
$$

Proof. We begin by manipulating the generating function for $p(n, A)$,

$$
\sum_{n=0}^{\infty} p(n, A) q^{n}=\prod_{i \in A} \frac{1}{\left(1-q^{i}\right)} \cdot \frac{E_{A}(q)}{E_{A}(q)}=\frac{E_{A}(q)}{\left(1-q^{\mathrm{lcm}(A)}\right)^{d}}=E_{A}(q) \sum_{k=0}^{\infty}\left(\begin{array}{c}
k+(d-1) \\
d-1
\end{array}\right) q^{\operatorname{lcm}(A) k}
$$

by line (11) and substituting $q$ with $q^{\mathrm{lcm}(A)}$ in $(12)$. Consider $n(\bmod \operatorname{lcm}(A))$, that is $n=\operatorname{lcm}(A) k+j$ for some positive integers $k$ and $j$. We consider the possibilities for arriving at an exponent of $q$ which is $\operatorname{lcm}(A) k+j$ in

$$
E_{A}(q) \sum_{k=0}^{\infty}\left(\begin{array}{c}
k+(d-1) \\
d-1
\end{array}\right) q^{\operatorname{lcm}(A) k}=\left(\begin{array}{c}
d \operatorname{lcm}(A)-\sum_{i \in A} i \\
\sum_{x=0} a_{x} q^{x}
\end{array}\right)\left(\sum_{k=0}^{\infty}\left(\begin{array}{c}
k+(d-1) \\
d-1
\end{array}\right) q^{\operatorname{lcm}(A) k}\right)
$$

which is exactly when $x+\operatorname{lcm}(A)(k-t)=\operatorname{lcm}(A) k+j$ and hence we have

$$
p(\operatorname{lcm}(A) k+j, A)=\sum_{t \geqslant 0} a_{j+\operatorname{lcm}(A) t}\left(\begin{array}{c}
k-t+(d-1) \\
d-1
\end{array}\right) .
$$

We arrive at a quasipolynomial for $p(n, A)$ when all values possible values of $j$ are considered in Theorem 5 are considered. In the case of $p(n, m), E_{m}(q)$ has a nice form $E_{m}(q)=\frac{\left(1-q^{\mathrm{lcm}(m)}\right)^{m}}{(q ; q)_{m}}$. For example, let $m=3$ then, 


$$
\begin{aligned}
E_{3}(q) & =\frac{\left(1-q^{6}\right)^{3}}{(q ; q)_{3}}=\left(1+q+q^{2}+q^{3}+q^{4}+q^{5}\right)\left(1+q^{2}+q^{4}\right)\left(1+q^{3}\right) \\
& =1+q+2 q^{2}+3 q^{3}+4 q^{4}+5 q^{5}+4 q^{6}+5 q^{7}+4 q^{8}+3 q^{9}+2 q^{10}+q^{11}+q^{12}
\end{aligned}
$$

Therefore, as a consequence of Theorem 5 , for $k \geqslant 0$,

$$
p(n, 3)= \begin{cases}p(6 k+0,3)=1\left(\begin{array}{c}
k+2 \\
2
\end{array}\right)+4\left(\begin{array}{c}
k+1 \\
2
\end{array}\right)+1\left(\begin{array}{c}
k \\
2
\end{array}\right) & =3 k^{2}+3 k+1 \\
p(6 k+1,3)=1\left(\begin{array}{c}
k+2 \\
2
\end{array}\right)+5\left(\begin{array}{c}
k+1 \\
2
\end{array}\right) & =3 k^{2}+4 k+1 \\
p(6 k+2,3)=2\left(\begin{array}{c}
k+2 \\
2
\end{array}\right)+4\left(\begin{array}{c}
k+1 \\
2
\end{array}\right) & =3 k^{2}+5 k+2 \\
p(6 k+3,3)=3\left(\begin{array}{c}
k+2 \\
2
\end{array}\right)+3\left(\begin{array}{c}
k+1 \\
2
\end{array}\right) & =3 k^{2}+6 k+3 \\
p(6 k+4,3)=4\left(\begin{array}{c}
k+2 \\
2
\end{array}\right)+2\left(\begin{array}{c}
k+1 \\
2
\end{array}\right) & =3 k^{2}+7 k+4 \\
p(6 k+5,3)=5\left(\begin{array}{c}
k+2 \\
2
\end{array}\right)+1\left(\begin{array}{c}
k+1 \\
2
\end{array}\right) & =3 k^{2}+8 k+5 .\end{cases}
$$

There are other quasipolynomoial expressions for $p(n, 3)$ such as $\left\|\frac{1}{12}(n+3)^{2}\right\|$, where $\| \cdot \mid$ denotes the nearest integer. Further explicit quasipolynomials for $p(n, 4), p(n, 5)$ and $p(n, 6)$ can be found in [13].

\subsection{A combinatorial interpretation of a quasipolynomial for $p(n, m)$}

Choliy and Sills in [7] provide a formula for $p(n)$ that "counts" using Durfee squares. Following their work, we aim to provide an analogous proof for the quasipolynomial formula of $p(n, m)$ in Theorem 5 that "counts". We start with essential definitions.

Recall that the notation $\lambda=\left(a_{1}, a_{2}, \ldots, a_{n}\right)$ where the $i^{\text {th }}$ component denotes the number of parts of size $i$ is used throughout. A subpartition of $\lambda$ is a partition such that $a_{i}^{\prime} \leqslant a_{i}$ for all parts of size $i$ and is denoted $\lambda^{\prime}=\left(a_{1}^{\prime}, a_{2}^{\prime}, \ldots, a_{k}^{\prime}\right)$. We say that two partitions $\lambda^{\prime}$ and $\lambda^{\prime \prime}$ sum to a partition $\lambda$ if for all $i, a_{i}=a_{i}^{\prime}+a_{i}^{\prime \prime}$ and is denoted $\lambda=\lambda^{\prime}+\lambda^{\prime \prime}$. Choliy and Sills [7] refer to this sum as the union of two partitions. We define remainder-like objects for $p(n, m)$.

Definition 6. A $\operatorname{lcm}(m)$-remainder partition is a partition such that there are no parts larger than $m$ and for any part of size $i$, there are less than $\frac{\operatorname{lcm}(m)}{i}$ copies of that part. Let $E_{m}$ be the collection of all $\operatorname{lcm}(m)$-remainder partitions. The generating function for $\operatorname{lcm}(m)$-remainder partitions is $E_{m}(q)$.

Example 7. A lcm(3)-remainder partition is $3+2+1=(1,1,1)$ but $3+3+1=(1,0,2)$ is not an lcm(3)-remainder partition.

Definition 8. Let the set of partitions $E_{m_{j}}$ be the partitions in $E_{m}$ such that they partition $j(\bmod \operatorname{lcm}(m))$.

Example 9. We give the example for $m=3$ and $j=0$. We have the set of partitions

$$
E_{3_{0}}=\{(0,0,0),(1,1,1),(2,2,0),(3,0,1),(4,1,0),(5,2,1)\} .
$$


Let $x$ be a non-negative integer, we say $a_{x}$ is the number of partitions in $E_{m}$ such that they partition $x$. We note that $a_{x}$ is the $x^{\text {th }}$ coefficient of $E_{m}(q)$. We now present a proof that "counts" the case of Theorem 5 when $A=\{1,2, \ldots, m\}$.

Theorem 10. For all $k \geqslant 0$ and $0 \leqslant j<l c m(m)$,

$$
p(l c m(m) k+j, m)=\sum_{t \geqslant 0} a_{l c m(m) t+j}\left(\begin{array}{c}
k-t+m-1 \\
m-1
\end{array}\right) .
$$

Proof. It is sufficient to consider partitions of $n$ into parts no larger than $m$. Let $n=$ $\operatorname{lcm}(m) k+j$ and consider a partition $\lambda=\left(a_{1}, a_{2}, \ldots, a_{m}\right)$. Let $r_{i} \equiv a_{i}\left(\bmod \frac{\operatorname{lcm}(m)}{i}\right)$. Define $\lambda_{r}=\left(r_{1}, \ldots, r_{m}\right)$ and $\lambda_{q}=\left(a_{1}-r_{1}, \ldots, a_{m}-r_{m}\right)$. Then we note first, $\lambda=\lambda_{q}+\lambda_{r}$. Second, $\lambda_{r} \in E_{m}$, and third, for any $i, \frac{\operatorname{lcm}(m)}{i}$ divides $a_{i}-r_{i}$, that is, $\frac{\operatorname{lcm}(m)}{i} k_{i}=a_{i}-r_{i}$ for some positive integer $k_{i}$. We will show that $\lambda_{r} \in E_{m_{j}}$ and that $k-t=\sum_{i=1}^{m} k_{i}$ for some non-negative integer $t$.

We start by showing $\lambda_{r} \in E_{m_{j}}$. Since $\lambda \vdash \operatorname{lcm}(m) k+j$, we have

$$
\operatorname{lcm}(m) k+j=\sum_{i=1}^{m} i a_{i}=\sum_{i=1}^{m} i\left(a_{i}-r_{i}\right)+\sum_{i=1}^{m} i r_{i}=\sum_{i=1}^{m} i \frac{\operatorname{lcm}(m)}{i} k_{i}+\sum_{i=1}^{m} i r_{i} .
$$

Therefore,

$$
\operatorname{lcm}(m) k+j \equiv \sum_{i=1}^{m} i r_{i} \equiv j \quad(\bmod \operatorname{lcm}(m))
$$

As $\sum_{i=1}^{m} i r_{i}=\lambda_{r}$, and is a partition of a number that is equivalent to $j(\bmod \operatorname{lcm}(m))$, then $\lambda_{r} \in E_{m_{r}}$ as desired. Express $\sum_{i=1}^{m} i r_{i}$ as $\operatorname{lcm}(m) t+j$ for some non-negative integer $t$. Furthermore, $\lambda_{q} \vdash \operatorname{lcm}(m)(k-t)$ by combining lines (20) and (21). Hence, by canceling $\operatorname{lcm}(m)$, we have $k-t=\sum_{i=1}^{m} k_{i}$ as desired.

Now, we count the partitions of $\operatorname{lcm}(m) k+j$ into parts no larger than $m$ in the following manner. We count the number of partitions such that $\lambda=\lambda_{q}+\lambda_{r}$ where $\lambda_{r} \vdash \operatorname{lcm}(m) t+j$ and $\lambda_{q} \vdash \operatorname{lcm}(m)(k-t)$. The number of choices for $\lambda_{r}$ is $a_{\operatorname{lcm}(m) t+j}$ since this is the number of partitions in $E_{m}$ and in $E_{m_{j}}$ such that they partition $\operatorname{lcm}(m) t+j$. By line (20) and Stars and Bars counting, the number of choices for $\lambda_{q} \vdash \operatorname{lcm}(m)(k-t)$ which is $\left(\begin{array}{c}k-t+m-1 \\ m-1\end{array}\right)$. Thus the number of choices for $\lambda$ is the product of the number of choices for $\lambda_{q}$ and $\lambda_{r}$. Accounting for every possibility of $t$, we have

$$
p(\operatorname{lcm}(m) k+j, m)=\sum_{t \geqslant 0} a_{\operatorname{lcm}(m) t+j}\left(\begin{array}{c}
k-t+m-1 \\
m-1
\end{array}\right) .
$$




\section{Partition identities for differences of partitions of $n$ into at most $m$ parts}

With a quasipolynomial for $p(n, m)$ in hand, we now aim to prove Theorem 3 and a new proof of Proposition 1.

Definition 11. We define the difference at distance $k$ of $p(n, m)$, denoted $\Delta_{k}(n, m)$, is

$$
\sum_{n=0}^{\infty} \Delta(n, m) q^{n}=\sum_{n=0}^{\infty}(p(n, m)-p(n-1, m)) q^{n}=\frac{1-q}{(q ; q)_{m}} .
$$

When $k \leqslant m, \Delta_{k}(n, m)$ can be interpreted as partitions of $n$ with at most $m$ parts without any parts of size $k$. In particular, these are twin partitions following [1] and we omit the subscript. When $A=\{1,2, \ldots, m\} \backslash 1$, then $\Delta(n, m)=p(n, A)$. In this case we say $p(n, A)=p(n, m \backslash 1)$ and use the notation $m \backslash 1$ in regards to $E_{A}$. Proposition 1 studies the difference of $p(n, m)-p(n-m, m)$ and Theorem 3 studies the first differences of $p(n, m)$.

The case of $m=4$ of Theorem 3 occurs in [1] and [6]. We provide a new proof for this via generating functions distinguishing the proof from the direct computation of differences of quasipolynomials as in [6] and the recursion used in [1]. It illustrates a necessary lemma to prove Theorem 3. Then we provide an example of the case of $m=5$ to build intuition for the arithmetic of Theorem 3. Results similar to the case of $m=3$ are found in articles regarding Gaussian polynomials such as in [6], [10], [11], and [12].

Theorem 12. For $\ell \geqslant 0$,

$$
\begin{aligned}
& p(2 \ell-3,4)-p(2 \ell-4,4)=p(\ell-3,3) \\
& p(2 \ell-4,4)-p(2 \ell-5,4)=p(\ell-2,3) .
\end{aligned}
$$

Proof. By considering $\ell$ modulo 6 in lines (24) and (25), the following is an equivalent set of statements: for $k \geqslant 0$,

$$
\begin{aligned}
p(6 k, 3) & =\left\{\begin{array}{l}
p(12 k, 4)-p(12 k-1,4) \\
p(12 k+3,4)-p(12 k+2,4)
\end{array}\right. \\
p(6 k+1,3) & =\left\{\begin{array}{l}
p(12 k+2,4)-p(12 k+1,4) \\
p(12 k+5,4)-p(12 k+4,4)
\end{array}\right. \\
p(6 k+2,3) & =\left\{\begin{array}{l}
p(12 k+4,4)-p(12 k+3,4) \\
p(12 k+7,4)-p(12 k+6,4)
\end{array}\right. \\
p(6 k+3,3) & =\left\{\begin{array}{l}
p(12 k+6,4)-p(12 k+5,4) \\
p(12 k+9,4)-p(12 k+8,4)
\end{array}\right. \\
p(6 k+4,3) & =\left\{\begin{array}{l}
p(12 k+8,4)-p(12 k+7,4) \\
p(12 k+11,4)-p(12 k+10,4)
\end{array}\right.
\end{aligned}
$$




$$
p(6 k+5,3)=\left\{\begin{array}{l}
p(12 k+10,4)-p(12 k+9,4) \\
p(12 k+13,4)-p(12 k+12,4)
\end{array} .\right.
$$

We following the proof of Theorem 5 we produce a quasipolynomial for $\Delta(n, 4)$ and $p(n, 3)$. Line (17) gives $E_{3}(q)$. Next, we must compute $E_{4 \backslash 1}(q)$ which is,

$$
\begin{gathered}
E_{4 \backslash 1}(q)=1+q^{2}+q^{3}+2 q^{4}+q^{5}+3 q^{6}+2 q^{7}+4 q^{8}+3 q^{9}+5 q^{10}+4 q^{11}+4 q^{12}+5 q^{13}+5 q^{14} \\
+4 q^{15}+4 q^{16}+5 q^{17}+3 q^{18}+4 q^{19}+2 q^{20}+3 q^{21}+q^{22}+2 q^{23}+q^{24}+q^{25}+q^{27} .
\end{gathered}
$$

We highlight that,

$$
\begin{array}{r}
E_{4 \backslash 1}(q)=\left(1+q^{3}\right)\left(1+q^{2}+2 q^{4}+3 q^{6}+4 q^{8}+5 q^{10}+4 q^{12}+5 q^{14}+4 q^{16}+3 q^{18}+2 q^{20}+q^{22}+q^{24}\right) \\
=\left(1+q^{3}\right) E_{3}\left(q^{2}\right) .
\end{array}
$$

Next, generating function arithmetic yields

$$
\begin{aligned}
\sum_{n=0}^{\infty} \Delta(n, 4) q^{n} & =\frac{1}{\left(q^{2} ; q\right)_{3}}=\frac{E_{4 \backslash 1}(q)}{E_{4 \backslash 1}(q)\left(q^{2} ; q\right)_{3}}=\frac{E_{4 \backslash 1}(q)}{\left(1-q^{12}\right)^{3}}=E_{4 \backslash 1}(q) \sum_{k=0}^{\infty}\left(\begin{array}{c}
k+2 \\
2
\end{array}\right) q^{12 k} \\
& =\left(1+q^{3}\right) E_{3}\left(q^{2}\right) \sum_{k=0}^{\infty}\left(\begin{array}{c}
k+2 \\
2
\end{array}\right) q^{12 k} \\
& =\left(1+q^{3}\right)\left(\sum_{k=0}^{\infty}\left(\left(\begin{array}{c}
k+2 \\
2
\end{array}\right)+4\left(\begin{array}{c}
k+1 \\
2
\end{array}\right)+\left(\begin{array}{c}
k \\
2
\end{array}\right)\right) q^{12 k}\right. \\
& +\sum_{k=0}^{\infty}\left(\left(\begin{array}{c}
k+2 \\
2
\end{array}\right)+5\left(\begin{array}{c}
k+1 \\
2
\end{array}\right)\right) q^{12 k+2} \\
& +\sum_{k=0}^{\infty}\left(2\left(\begin{array}{c}
k+2 \\
2
\end{array}\right)+4\left(\begin{array}{c}
k+1 \\
2
\end{array}\right)\right) q^{12 k+4}+\sum_{k=0}^{\infty}\left(3\left(\begin{array}{c}
k+2 \\
2
\end{array}\right)+3\left(\begin{array}{c}
k+1 \\
2
\end{array}\right)\right) q^{12 k+6} \\
& \left.\left.\left.+\sum_{k=0}^{\infty}\left(\begin{array}{c}
k+2 \\
2
\end{array}\right)+2\left(\begin{array}{c}
k+1 \\
2
\end{array}\right)\right) q^{12 k+8}+\sum_{k=0}^{\infty}\left(\begin{array}{c}
k+2 \\
2
\end{array}\right)+\left(\begin{array}{c}
k+1 \\
2
\end{array}\right)\right) q^{12 k+10}\right) \\
& =\left(1+q^{3}\right)\left(\begin{array}{c}
\infty \\
\sum_{k=0}^{\infty} p(6 k, 3) q^{12 k}+\sum_{k=0}^{\infty} p(6 k+1,3) q^{12 k+2}+\sum_{k=0}^{\infty} p(6 k+2,3) q^{12 k+4} \\
\end{array}\right. \\
& \left.+\sum_{k=0}^{\infty} p(6 k+3,3) q^{12 k+6}+\sum_{k=0}^{\infty} p(6 k+4,3) q^{12 k+8}+\sum_{k=0}^{\infty} p(6 k+4,3) q^{12 k+10}\right) .
\end{aligned}
$$

by lines (17), (18), and (26). Comparing powers of $q$ modulo 12 in the preceding equation, the result is as desired.

The ability to factor $E_{4 \backslash 1}(q)$ into $\left(1+q^{3}\right) E_{3}\left(q^{2}\right)$ was essential in proving the claim. Fortunately, in this specific case a computer can handle this factorization with ease as $E_{4 \backslash 1}(q)$ is a polynomial of degree 27 . This leads to the following lemma which states under 
what conditions $E_{m \backslash 1}(q)$ factors. We will provide three proofs for. The first two proofs will occur in this section; one following the generating function arithmetic of Theorem 5 and another following the counting of Theorem 10. The third occurs in Section 4.

Lemma 13. Let $m=s^{k}$ where $s$ is prime and $k$ is a positive integer, then $E_{m \backslash 1}(q)=$ $G(q) E_{m-1}\left(q^{s}\right)$ where $G(q)=\prod_{i=2, i \neq s^{a}, \forall a \in \mathbb{N}}^{m} \sum_{j=0}^{s-1} q^{i j}$.

Remark 14. Strictly speaking, $G(q)$ is the generating function for the collection of $1 \mathrm{~cm}(m)$ remainder partitions which have no parts of size 1 , any positive integer power of $s$, and parts of any size occur less than $s$ times. The nature of $G(q)$ is seemly mysterious. In a broader context, we can also think of $G(q)$ as the generating function for a collection of "remainder partitions" when the collection of partitions generated by $E_{m \backslash 1}(q)$ are considered modulo $\operatorname{lcm}(m-1)$-remainder partitions. This arises naturally from defining a group structure on partitions generated by $E_{A}(q)$ which will be treated in Section 4 . For now, we can experience $G(q)$ as a consequence of arithmetic and as a property of the sum of partitions and some particular combinatorial map.

Proof. We show that $\frac{E_{m \backslash 1}(q)}{E_{m-1}(q)}=G(q)$ by simplifying the expression,

$$
\begin{array}{r}
\frac{E_{m \backslash 1}(q)}{E_{m-1}\left(q^{s}\right)}=\frac{\frac{\left(1-q^{\operatorname{lcm}(m)}\right)^{m-1}}{\left(q^{2} ; q\right)_{m-1}}}{\frac{\left(1-q^{\operatorname{lcm}(m)}\right)^{m-1}}{\left(q^{s} ; q^{s}\right)_{m-1}}}=\frac{\left(q^{s} ; q^{s}\right)_{m-1}}{\left(q^{2} ; q\right)_{m-1}}=\frac{\prod_{r=1}^{k}\left(\left(1-q^{s^{r}}\right) \prod_{i=s^{r-1}+1}^{s^{k}-1}\left(1-q^{s i}\right)\right)}{\prod_{r=1}^{k}\left(\left(1-q^{s^{r}}\right) \prod_{i=s^{r-1}+1}^{s^{k}-1}\left(1-q^{i}\right)\right)} \\
=\frac{\prod_{r=1}^{k} \prod_{i=s^{r-1}+1}^{s^{k}-1}\left(\sum_{j=0}^{s-1} q^{i j}\right)\left(1-q^{i}\right)}{\prod_{r=1}^{k} \prod_{i=s^{r-1}+1}^{s^{k}-1}\left(1-q^{i}\right)}=G(q) .
\end{array}
$$

For the combinatorial proof of Lemma 13, we introduce a generalization of $\operatorname{lcm}(m)$ remainder partitions. Furthermore, we discuss how the sums of restricted partitions behave in regards to their generating functions. Definition 6 can be generalized by using a finite set positive integers $A$ in place $m$. The notation uses $A$ in place of $m$ with as opposed to each part form 1 though $m$, we have parts from the set $A$.

Proposition 15. Let'," , and '"' be restrictions on partitions such that for any $\lambda^{\prime \prime}$ and $\lambda^{\prime \prime \prime}$ obeying " and "' respectively, that the sum of $\lambda^{\prime \prime}$ and $\lambda^{\prime \prime \prime}$ is a partition, $\lambda^{\prime}$, obeying '. If there are unique partitions $\lambda^{\prime \prime}$ and $\lambda^{\prime \prime \prime}$ such that $\lambda^{\prime}=\lambda^{\prime \prime}+\lambda^{\prime \prime \prime}$, then

$$
\sum_{n \geqslant 0} p^{\prime}(n) q^{n}=\left(\sum_{n \geqslant 0} p^{\prime \prime}(n) q^{n}\right)\left(\sum_{n \geqslant 0} p^{\prime \prime \prime}(n) q^{n}\right) .
$$

Proof. Suppose that there are unique partitions $\lambda^{\prime \prime}$ and $\lambda^{\prime \prime \prime}$ such that $\lambda^{\prime}=\lambda^{\prime \prime}+\lambda^{\prime \prime \prime}$. Let $n$ be given, we work on counting the number of partitions of $n$ such that they obey the 
restriction '. First, for any $0 \leqslant i \leqslant n, p^{\prime \prime}(i) p^{\prime \prime \prime}(n-i)$ is the number of ways such that if $\lambda^{\prime \prime} \vdash i$ and $\lambda^{\prime \prime \prime} \vdash n-i$ then $\lambda^{\prime \prime}+\lambda^{\prime \prime \prime} \vdash n$ where $\lambda^{\prime \prime}+\lambda^{\prime \prime \prime}$ is a partition that satisfies the restrictions '. Then over all possibilities of $i$, this yields $p^{\prime}(n)=\sum_{i=0}^{n} p^{\prime \prime}(i) p^{\prime \prime \prime}(n-i)$ as the sums are assumed to be unique, no partition is counted twice and there is never a partition satisfying the restriction ' such that there are no $\lambda^{\prime \prime}$ and $\lambda^{\prime \prime \prime}$ that sum to it. We now observe the product of the generating functions of partitions of $n$ satisfying " and "'I respectively. We arrive at

$$
\left(\sum_{n \geqslant 0} p^{\prime \prime}(n) q^{n}\right)\left(\sum_{n \geqslant 0} p^{\prime \prime \prime}(n) q^{n}\right)=\sum_{n \geqslant 0}\left(\sum_{i=0}^{n} p^{\prime \prime}(i) p^{\prime \prime \prime}(n-i)\right) q^{n}=\sum_{n \geqslant 0} p^{\prime}(n) q^{n} .
$$

We now provide a combinatorial proof of Lemma 13.

Proof. Let $\lambda_{\backslash 1}=\left(0, a_{2}, \ldots, a_{m}\right) \in E_{m \backslash 1}$. Let $\lambda_{\backslash 1}^{(r)}=\left(0, r_{2}, \ldots, r_{m}\right)$ be a sub partition of $\lambda_{\backslash 1} . \lambda_{1}^{(r)}$ is defined by two cases, parts that are powers of $s$ and parts that are not powers of $s$. If a part of size $i$ is a power of $s$, then the number of parts $r_{i}$, is zero. Otherwise, we define the number of parts, $r_{i}=\equiv a_{i}(\bmod s)$. Let $\lambda_{\backslash 1}^{(q)}$ be $\left(0, a_{2}-r_{2}, \ldots, a_{m}-r_{m}\right)$. The number $a_{i}-r_{i}$, unless $i$ is a power of $s$, is divisible by $s$. We have $\lambda_{\backslash 1}=\lambda_{\backslash 1}^{(r)}+$ $\lambda_{\backslash 1}^{(q)}$. Furthermore, by the uniqueness of quotient and remainders of non-negative integer division, this sum is unique.

$\lambda_{\backslash 1}^{(r)}$ is a partition generated $G(q)$. That is, $\lambda_{\backslash 1}^{(r)}$ is a partition such that no part has a power of $s$ and for any part of size $i$ there are less than $s$ copies. The generating function $E_{m-1}\left(q^{s}\right)$ describes partitions such parts of size $i$ that are powers of $s$ occur no more than $\operatorname{lcm}(m-1)=\frac{\operatorname{lcm}(m)}{s}$ times and parts of size $i$ that are not powers of $s$ occur a multiple of $s$ number of times, with no more than $\frac{s \cdot \mathrm{lcm}(m-1)}{i}$ copies of a part of size $i$. Any subpartition $\lambda_{\backslash 1}^{(q)}$ if $\lambda_{\backslash 1}$ is generated by $E_{m-1}\left(q^{s}\right)$ as its construction satisfies the description of partitions generated by $E_{m-1}\left(q^{s}\right)$.

Let ' be the restriction describing partitions in $E_{m \backslash 1}$. Let " be the restriction that is described by $\lambda_{\backslash 1}^{(r)}$ in the first paragraph of this proof and "' be the restriction that is described by $\lambda_{\backslash 1}^{(q)}$ in the first paragraph. The restrictions ',", ,"' satisfy the conditions of Proposition 15, and the lemma is proven.

With Lemma 13 in hand, deducing if a constituent of $\Delta(n, m)$ can be expressed as a non-negative linear combination of constituents of $p(n, m-1)$ is straightforward.

Example 16. Let $m=5$, then by Lemma 13,

$$
E_{5 \backslash 1}(q)=G(q) E_{4}\left(q^{5}\right)=\left(\prod_{i=2}^{4} \sum_{j=0}^{4} q^{i j}\right) E_{4}\left(q^{5}\right) .
$$


Now, construct the respective quasipolynomials of $\Delta(n, 5)$ and $p(n, 4)$,

$$
\begin{aligned}
& \sum_{n=0}^{\infty} \Delta(n, 5) q^{n}=\frac{1}{\left(q^{2} ; q\right)_{4}}=\frac{E_{5 \backslash 1}(q)}{\left(1-q^{60}\right)^{4}}=E_{5 \backslash 1}(q) \sum_{k=0}^{\infty}\left(\begin{array}{c}
k+3 \\
3
\end{array}\right) q^{60 k} \\
&=G(q) E_{4}\left(q^{5}\right) \sum_{k=0}^{\infty}\left(\begin{array}{c}
k+3 \\
3
\end{array}\right) q^{60 k}=\left(\prod_{i=2}^{4} \sum_{j=0}^{4} q^{i j}\right) E_{4}\left(q^{5}\right) \sum_{k=0}^{\infty}\left(\begin{array}{c}
k+3 \\
3
\end{array}\right) q^{60 k} \\
& \text { and } \sum_{n=0}^{\infty} p(n, 4) q^{n}=\frac{1}{(q ; q)_{4}}=\frac{E_{4}(q)}{\left(1-q^{12}\right)^{4}}=E_{4}(q) \sum_{k=0}^{\infty}\left(\begin{array}{c}
k+3 \\
3
\end{array}\right) q^{12 k}
\end{aligned}
$$

Where

$$
\begin{gathered}
G(q)=\left(\prod_{i=2}^{4} \sum_{j=0}^{4} q^{i j}\right)=1+q^{2}+q^{3}+2 q^{4}+q^{5}+3 q^{6}+2 q^{7}+4 q^{8}+3 q^{9}+4 q^{10}+4 q^{11}+6 q^{12} \\
+4 q^{13}+6 q^{14}+5 q^{15}+7 q^{16}+5 q^{17}+7 q^{18}+5 q^{19}+7 q^{20}+5 q^{21}+6 q^{22}+4 q^{23}+6 q^{24}+4 q^{25}+4 q^{26}+3 q^{27} \\
+4 q^{28}+2 q^{29}+3 q^{30}+q^{31}+2 q^{32}+q^{33}+q^{34}+q^{36}, \quad(34) \\
E_{4}(q)=1+q+2 q^{2}+3 q^{3}+5 q^{4}+6 q^{5}+9 q^{6}+11 q^{7}+15 q^{8}+18 q^{9}+23 q^{10}+27 q^{11}+30 q^{12}+35 q^{13} \\
+39 q^{14}+42 q^{15}+44 q^{16}+48 q^{17}+48 q^{18}+50 q^{19}+48 q^{20}+48 q^{21}+44 q^{22}+42 q^{23}+39 q^{24}+35 q^{25} \\
+30 q^{26}+27 q^{27}+23 q^{28}+18 q^{29}+15 q^{30}+11 q^{31}+9 q^{32}+6 q^{33}+5 q^{34}+3 q^{35}+2 q^{36}+q^{37}+q^{38}, \text { and }
\end{gathered}
$$

$$
\begin{array}{r}
E_{4}\left(q^{5}\right)=1+q^{5}+2 q^{10}+3 q^{15}+5 q^{20}+6 q^{25}+9 q^{30}+11 q^{35}+15 q^{40}+18 q^{45}+23 q^{50}+27 q^{55}+30 q^{60} \\
+35 q^{65}+39 q^{70}+42 q^{75}+44 q^{80}+48 q^{85}+48 q^{90}+50 q^{95}+48 q^{100}+48 q^{105}+44 q^{110}+42 q^{115}+39 q^{120} \\
+35 q^{125}+30 q^{130}+27 q^{135}+23 q^{140}+18 q^{145}+15 q^{150}+11 q^{155}+9 q^{160}+6 q^{165}+5 q^{170}+3 q^{175}+2 q^{180} \\
+q^{185}+q^{190} .
\end{array}
$$

For example, $\Delta(60 k+0,5)=p(12 k+0,4)+p(12 k-1,4)+4 p(12 k-2,4)+5 p(12 k-$ $3,4)+7 p(12 k-4,4)+4 p(12 k-5,4)+3 p(12 k-6,4)$.

While further arithmetic could explicitly show the partition identity between $\Delta(n, 5)$ and $p(n, 4)$, it is tedious and the observed relationship above motivates the proof of the Theorem 3. We now prove Theorem 3 .

Proof. We manipulate the generating function of $\Delta(n, m)$ following the proof for Theorem 5 and apply Lemma 13,

$$
\sum_{n=0}^{\infty} \Delta(n, m) q^{n}=\frac{1}{\left(q^{2} ; q\right)_{m-1}}=\frac{E_{m \backslash 1}(q)}{E_{m \backslash 1}(q)\left(q^{2} ; q\right)_{m-1}}=\frac{E_{m \backslash 1}(q)}{\left(1-q^{\operatorname{lcm}(m)}\right)^{m-1}}
$$




$$
=E_{m \backslash 1}(q) \sum_{k=0}^{\infty}\left(\begin{array}{c}
k+m-2 \\
m-2
\end{array}\right) q^{\operatorname{lcm}(m) k}=G(q) E_{m-1}\left(q^{s}\right) \sum_{k=0}^{\infty}\left(\begin{array}{c}
k+m-2 \\
m-2
\end{array}\right) q^{\operatorname{lcm}(m) k} .
$$

with $G(q)=\prod_{i=2, i \neq s^{a}, \forall a \in \mathbb{N}}^{m} \sum_{j=0}^{s-1} q^{i j}$. Next, we construct the quasipolynomial for $p(n, m-1)$,

$$
\sum_{n=0}^{\infty} p(n, m-1) q^{n}=\frac{1}{(q ; q)_{m-1}}=\frac{E_{m-1}(q)}{\left(1-q^{\mathrm{lcm}(m-1)}\right)^{m-1}}=E_{m-1}(q) \sum_{k=0}^{\infty}\left(\begin{array}{c}
k+m-2 \\
m-2
\end{array}\right) q^{\mathrm{lcm}(m-1) k} .
$$

Express the polynomials $E_{m \backslash 1}(q), E_{m-1}(q)$, and $G(q)$ as

$$
E_{m \backslash 1}(q)=\sum_{i \geqslant 0} a_{i} q^{i}, E_{m-1}(q)=\sum_{i \geqslant 0} b_{i} q^{i}, \text { and } G(q)=\sum_{i \geqslant 0} c_{i} q^{i} .
$$

Notice if the index of any coefficient of $E_{m \backslash 1}(q), E_{m-1}(q)$, and $G(q)$ is less than zero, then the coefficient is zero. For any constituent

$$
\Delta(\operatorname{lcm}(m) k+j, n)=\sum_{t \geqslant 0} a_{j+\operatorname{lcm}(m) t}\left(\begin{array}{c}
k+m-2-t \\
m-2
\end{array}\right)
$$

of the quasipolynomial of $\Delta(n, m)$, let $l$ and $r$ be integers such that $j=l+r$ where $r \equiv j$ $(\bmod s)$ with $0 \leqslant r<s$. Since $G(q) E_{m-1}\left(q^{s}\right)=E_{m \backslash 1}(q)$ by Lemma 13 , this implies by polynomial multiplication that the coefficient $a_{j+\operatorname{lcm}(m) t}$ in line (41) is

$$
a_{j+\operatorname{lcm}(m) t}=\sum_{i \geqslant 0} c_{i s+r} b_{\operatorname{lcm}(m) t+l-i s} .
$$

Therefore, we apply line (42) to line (41) and rearrange the sum to see

$$
\begin{aligned}
& \sum_{t \geqslant 0} a_{j+\operatorname{lcm}(m) t}\left(\begin{array}{c}
k+m-2-t \\
m-2
\end{array}\right)=\sum_{t \geqslant 0} \sum_{i \geqslant 0} c_{i s+r} b_{\operatorname{lcm}(m) t+l-i s}\left(\begin{array}{c}
k+m-2-t \\
m-2
\end{array}\right) \\
& =c_{r} \sum_{t \geqslant 0} b_{\operatorname{lcm}(m) t+l}\left(\begin{array}{c}
k+m-2-t \\
m-2
\end{array}\right)+\sum_{i \geqslant 1} \sum_{t \geqslant 0} c_{i s+r} b_{\operatorname{lcm}(m) t+l-i s}\left(\begin{array}{c}
k+m-2-t \\
m-2
\end{array}\right) \\
& =c_{r} \sum_{t \geqslant 0} b_{\operatorname{lcm}(m) t+l}\left(\begin{array}{c}
k+m-2-t \\
m-2
\end{array}\right)+\sum_{i \geqslant 1} \sum_{t \geqslant 1} c_{i s+r} b_{\operatorname{lcm}(m) t+l-i s}\left(\begin{array}{c}
k+m-2-t \\
m-2
\end{array}\right) \\
& =c_{r} \sum_{t \geqslant 0} b_{\operatorname{lcm}(m) t+l}\left(\begin{array}{c}
k+m-2-t \\
m-2
\end{array}\right)+\sum_{i \geqslant 1} c_{i s+r} \sum_{t \geqslant 0} b_{\operatorname{lcm}(m)(t-1)+l-i s}\left(\begin{array}{c}
k+m-2-(t+1) \\
m-2
\end{array}\right) .
\end{aligned}
$$

Set $l^{\prime}=\frac{l}{s}$. We now compare line $(46)$ to constituents of $p(n, m-1)$. In particular, $p\left(\operatorname{lcm}(m-1) k+l^{\prime}+(\operatorname{lcm}(m-1)-i), m-1\right)=\sum_{t \geqslant 0} b_{\operatorname{lcm}(m-1) t+l^{\prime}+(\operatorname{lcm}(m-1)-i)}\left(\begin{array}{c}k+m-2-t \\ m-2\end{array}\right)$. 
Taking $k=k-1$ we have,

$$
\begin{array}{r}
p\left(\operatorname{lcm}(m-1)(k-1)+l^{\prime}+(\operatorname{lcm}(m-1)-i), m-1\right)=p\left(\operatorname{lcm}(m-1) k+l^{\prime}-i, m-1\right) \\
=\sum_{t \geqslant 0} b_{\operatorname{lcm}(m-1) t+l^{\prime}+(\operatorname{lcm}(m-1)-i)}\left(\begin{array}{c}
k+m-2-(t+1) \\
m-2
\end{array}\right)
\end{array}
$$

By substituting $q$ for $q^{s}$ in $E_{m-1}(q)$, we have $b_{\operatorname{lcm}(m) t+l+i s}=b_{\operatorname{lcm}(m-1) t+l^{\prime}+i}$. Furthermore, since $\operatorname{lcm}(m)=s \cdot \operatorname{lcm}(m-1)$, the following holds by combining lines (46) and (48),

$$
\Delta(\operatorname{lcm}(m) k+j, m)=\sum_{i \geqslant 0} c_{r+s i} p\left(\operatorname{lcm}(m-1) k+l^{\prime}-i, m-1\right) .
$$

We now prove Proposition 1.

Proof. We start by creating the quasipolynomial for $\Delta(n, m)$ and apply properties of $E_{m}(q)$ and $E_{m \backslash 1}(q)$,

$$
\begin{aligned}
& \sum_{n=0}^{\infty} \Delta(n, m) q^{n}=\frac{1}{\left(q^{2} ; q\right)_{m-1}}=\frac{1-q}{(q ; q)_{m}}=\frac{(1-q) E_{m}(q)}{\left(1-q^{\operatorname{lcm}(m)}\right)^{m}} \\
& =\frac{E_{m \backslash 1}(q)}{\left(1-q^{\operatorname{lcm}(m)}\right)^{m-1}}=E_{m \backslash 1}(q) \sum_{k=0}^{\infty}\left(\begin{array}{c}
k+m-2 \\
m-2
\end{array}\right) q^{\operatorname{lcm}(m) k} .
\end{aligned}
$$

Next, we address $p(n, m-1)$.

$$
\begin{aligned}
& \sum_{n=0}^{\infty} p(n, m-1) q^{n}=\frac{1}{(q ; q)_{m-1}}=\frac{1-q^{m}}{(q ; q)_{m}}=\frac{\left(1-q^{m}\right) E_{m}(q)}{\left(1-q^{\mathrm{lcm}(m)}\right)^{m}} \\
& =\frac{(1-q)\left(\sum_{i=0}^{m-1} q^{i}\right) E_{m}(q)}{\left(1-q^{\mathrm{lcm}(m)}\right)^{m}}=\frac{\left(\sum_{i=0}^{m-1} q^{i}\right) E_{m \backslash 1}(q)}{\left(1-q^{\operatorname{lcm}(m)}\right)^{m-1}} \\
& =\left(\sum_{i=0}^{m-1} q^{i}\right) E_{m \backslash 1}(q) \sum_{k=0}^{\infty}\left(\begin{array}{c}
k+m-2 \\
m-2
\end{array}\right) q^{\operatorname{lcm}(m) k} .
\end{aligned}
$$

The arguments in the proof of Theorem 3 yield,

$$
p(\operatorname{lcm}(m-1) k+j, m-1)=\sum_{i=0}^{m-1} \Delta(\operatorname{lcm}(m) k+j-i, m)=\Delta_{m}(\operatorname{lcm}(m) k+j, m) .
$$

Thus $p(n, m-1)$ can always be written as a linear combination of constituents of $\Delta(n, m)$ in a manner that is equivalent to the familiar partition identity, $p(n, m-1)=p(n, m)-$ $p(n-m, m)$. 


\section{Partition identities for partitions with parts from Finite Sets $A$ and $B$}

We now aim to generalize the previous section into partitions with parts from finite sets $A$ and $B$. First, $\operatorname{lcm}(A)$-remainder partitions are equipped with a natural group structure as an operation on the number of parts.

Definition 17. Let $A$ be a finite set of positive integers and consider the set $E_{A}$ consisting of all $\operatorname{lcm}(A)$-remainder partitions. We define an operation, $\oplus$, on $E_{A}$. Let $\lambda_{1}, \lambda_{2} \in E_{A}$, and $\lambda_{1}=\left(a_{i_{1}, 1}, \ldots, a_{i_{k}, \alpha_{i_{k}}}\right)$ and $\lambda_{2}=\left(b_{i_{1}, 1}, \ldots, b_{i_{k}, \alpha_{i_{k}}}\right)$. Then $\lambda_{1} \oplus \lambda_{2}=\lambda_{3}=$ $\left(c_{i_{1}, 1}, \ldots, c_{i_{k}, \alpha_{i_{k}}}\right)$ by defining $c_{i_{j}, \ell}$ with $\ell \in\left\{1,2, \ldots, \alpha_{i_{j}}\right\}$ to be the smallest non-negative remainder of $a_{i_{j}, \ell}+b_{i_{j}, \ell}$ divided by $\frac{\operatorname{lcm}(A)}{i_{j}}$. That is, $a_{i_{j}, \ell}+b_{i_{j}, \ell} \equiv c_{i_{j}, \ell}\left(\bmod \frac{\operatorname{lcm}(A)}{i_{j}}\right)$. The operation $\oplus$ is called piecewise modular addition.

The operation $\oplus$ on $E_{A}$ is a commutative group. The notation $E_{A}$ will be used for both the group $\left(E_{A}, \oplus\right)$ and the set of partitions $\operatorname{lcm}(A)$-remainder partitions. We now give several important properties of $E_{A}$, the group of $\operatorname{lcm}(A)$-remainder partitions.

Proposition 18. The group $E_{A}$ is isomorphic to $\prod_{i \in A} \mathbb{Z}_{\frac{l c m(A)}{i}}$ where the product is the usual direct product of groups and $\mathbb{Z}_{n}$ is a cyclic group of order $n$.

The proof is left to the reader. The group $E_{A}$ has order $\prod_{i \in A} \frac{\operatorname{lcm}(A)}{i}$ by Proposition 18 or by counting and the order of any element in $E_{A}$ is at most $\operatorname{lcm}(A)$.

Proposition 19. Let $\lambda_{1}, \lambda_{2} \in E_{A}$ and suppose that $\lambda_{1} \vdash g$ and $\lambda_{2} \vdash h$. Then $\lambda_{1} \oplus \lambda_{2} \vdash$ $(g+h)(\bmod l c m(A))$.

Proof. We first note that $g=\sum_{s=1}^{k} \sum_{t=1}^{\alpha_{i_{s}}} i_{j} \cdot a_{i_{s}, t}$ and $h=\sum_{s=1}^{k} \sum_{t=1}^{\alpha_{i_{s}}} i_{j} \cdot b_{i_{s}, t}$. Furthermore, it must be the case that $\lambda_{1} \oplus \lambda_{2}$ implies that $\left(a_{i_{s}, t}+b_{i_{s}, t}\right)=c_{i_{s}, t}+x_{i_{s}, t}\left(\frac{\operatorname{lcm}(A)}{i_{s}}\right)$ for some positive integer $x_{i_{s}, t}$ by definition. The following arithmetic shows the result,

$$
\begin{aligned}
g+h & =\sum_{s=1}^{k} \sum_{t=1}^{\alpha_{i_{s}}} i_{j}\left(a_{i_{s}, t}+b_{i_{s}, t}\right) \\
& =\sum_{s=1}^{k} \sum_{t=1}^{\alpha_{i_{s}}} i_{j}\left(c_{i_{s}, t}+x_{i_{s}, t}\left(\frac{\operatorname{lcm}(A)}{i_{s}}\right)\right) \\
& =\sum_{s=1}^{k} \sum_{t=1}^{\alpha_{i_{s}}}\left(i_{j} c_{i_{s}, t}+x_{i_{s}, t} \operatorname{lcm}(A)\right) \\
& =\lambda_{1} \oplus \lambda_{2}+\operatorname{lcm}(A) \sum_{s=1}^{k} \sum_{t=1}^{\alpha_{i_{s}}} x_{i_{s}, t} \equiv \lambda_{1} \oplus \lambda_{2} \quad(\bmod \operatorname{lcm}(A)) .
\end{aligned}
$$


The proof of Proposition 19 closely follows the strategy employed in Theorem 10 applied strictly to $\operatorname{lcm}(A)$-remainder partitions. We are now ready to state and prove the generalization of Lemma 13.

Lemma 20. Let $A$ and $B$ be two sets of positive integers with $|B| \leqslant|A|$. If there is an onto homomorphism from $E_{A}$ to $E_{B}$, then $E_{A}(q)=G(q) E_{B}\left(q^{s}\right)$ for $G(q)$ a generating function of some collection of $l \mathrm{~cm}(A)$-remainder partitions and $s=\frac{\operatorname{lcm}(A)}{\operatorname{lcm}(B)}$.

Proof. Suppose that there is an onto homomorphism $\varphi$ from $E_{A}$ to $E_{B}$. By the first isomorphism theorem, $\operatorname{Im}(\varphi) \cong E_{A} / \operatorname{ker}(\varphi)$ and as the mapping is onto, $\operatorname{Im}(\varphi) \cong E_{B}$. We essentially have two tasks; first, show that there is a collection of partitions $H \subseteq E_{A}$ that is generated by $E_{B}\left(q^{s}\right)$; second, to find a collection, $G$, of $\operatorname{lcm}(A)$-remainder partitions such that $H$ and $G$ are restrictions of $\operatorname{lcm}(A)$-remainder partitions which satisfy the conditions of Proposition 15.

To satisfy the first task, we make a few notes about $E_{B}$ in relationship to $E_{A}$. Let $\lambda_{i_{v, w}}^{(A)} \in E_{A}$ be be a partition that has one part of size $i_{v}$ in color $w . \lambda_{i_{v, w}}^{(A)}$ is a generator of group $E_{A}$. Likewise, let $\lambda_{i_{t, u}}^{(B)} \in E_{B}$ be a partition that has one part of size $i_{t}$ in color $u$. Likewise $\lambda_{i_{t, u}}^{(B)}$ is a generator of the group $E_{B}$. As $\varphi$ can be viewed as an onto homomorphism for direct products of cyclic groups, it is the case that $\varphi\left(x \cdot \lambda_{i_{v, w}}^{(A)}\right)=\lambda_{i_{t}, u}^{(B)}$ where $x \cdot \lambda_{i_{v, w}}^{(A)}:=\bigoplus_{k=1}^{x} \lambda_{i_{v, w}}^{(A)}$ is piecewise modular addition of $\lambda_{i_{v, w}}^{(A)} x$ times and $\operatorname{gcd}\left(x, \frac{\operatorname{lcm}(A)}{i_{v}}\right)=1$. Since the order of $x \cdot \lambda_{i_{v, w}}^{(A)}$ is $\frac{\operatorname{lcm}(A)}{i_{v, w}}$, we have

$$
\sum_{k=1}^{\frac{\operatorname{lcm}(A)}{i_{v}, w}}\left(\sum_{j=1}^{x} \lambda_{i_{v, w}}^{(A)}\right) \vdash x \cdot i_{v, w} \cdot \frac{\operatorname{lcm}(A)}{i_{v, w}}=x \cdot \operatorname{lcm}(A)
$$

and in the group $E_{A}$, it is the case that $\frac{\operatorname{lcm}(A)}{i_{v, w}} \cdot\left(x \cdot \lambda_{i_{v, w}}^{(A)}\right)=\lambda_{e}$. We apply $\varphi$, which yields

$$
\frac{\operatorname{lcm}(A)}{i_{v, w}} \cdot \varphi\left(x \cdot \lambda_{i_{v, w}}^{(A)}\right)=\frac{\operatorname{lcm}(A)}{i_{v, w}} \cdot \lambda_{i_{t, u}}^{(B)}=\lambda_{e}^{(B)} .
$$

Applying Proposition 19 to (57), we see that

$$
\lambda_{e}^{(B)}=\bigoplus_{r=1}^{\frac{\operatorname{lcm}(A)}{i_{v}, w}} \lambda_{i_{t, u}}^{(B)} \vdash \sum_{r=1}^{\frac{\operatorname{lcm}(A)}{i_{v, w}}} i_{t, u}=\frac{\operatorname{lcm}(A)}{i_{v, w}} \cdot i_{t, u} \equiv 0 \quad(\bmod \operatorname{lcm}(B)) .
$$

This implies that there is a smallest positive integer $y$ such that

$$
y \cdot \operatorname{lcm}(B)=\frac{\operatorname{lcm}(A)}{i_{v, w}} \cdot i_{t, u} \text { which is equivalent to } y \cdot i_{v, w}=\frac{\operatorname{lcm}(A)}{\operatorname{lcm}(B)} \cdot i_{t, u}=s \cdot i_{t, u} .
$$

If $s \mid y$ in the previous line, there is a positive integer $z$ such that $z \cdot i_{v, w}=i_{t, u}$. Furthermore, $z \cdot i_{v, w} \cdot \frac{\operatorname{lcm}(B)}{i_{t, u}}=\operatorname{lcm}(A)$. If $s \not Y$ in line (59), then $s \mid i_{v, w}$. For each generator $\lambda_{i_{t, u}}^{(B)}$ of $E_{B}$, 
we name a partition $\lambda_{i_{t, u}}^{(A)} \in E_{A}$. Let $\lambda_{i_{t, u}}^{(A)}=y \cdot \lambda_{i_{v, w}}^{(A)} \in E_{A}$ such that $y \cdot \lambda_{i_{v, w}}^{(A)} \vdash s \cdot i_{t, u}$. We define the set $H$ by

$$
H=\left\{\bigoplus_{i_{t, u} \in B} k_{i_{t, u}} \cdot \lambda_{i_{t, u}}^{(A)} \mid 0 \leqslant k_{i_{t, u}}<\frac{\operatorname{lcm}(B)}{i_{t, u}}\right\}
$$

We have a natural bijection between $H$ and $E_{B}$ by mapping $\lambda_{i_{t, u}}^{(A)}$ to $\lambda_{i_{t, u}}^{(B)}$. Next, if $\lambda \vdash n$ in $E_{B}$, the corresponding partition by the natural bijection in $H$ must partition $s \cdot n$. By this correspondence, the generating function for $H$ must be $E_{B}\left(q^{s}\right)$.

With our first task complete, we turn to the second. Let $\lambda \in E_{A}$. We will define $\lambda_{q}$ and $\lambda_{r}$ such that $\lambda_{q}+\lambda_{r}=\lambda$ and that $\lambda_{q} \in H, \lambda_{r} \in G$. We start by defining $\lambda_{r}$. For the number $a_{i_{v, w}}$ of parts of size $i_{v}$ and color $w$, if there is a $\lambda_{i_{t, u}}^{(A)} \in H$ such that $\lambda_{i_{t, u}}^{(A)}=y \cdot \lambda_{i_{v, w}}^{(A)} \vdash s \cdot i_{t, u}$ then we have two cases: if $y \mid s$ and if $y \bigwedge s$. For the first case, we divide $a_{i_{v, w}}$ by $y$ letting the remainder be defined as $r_{i_{v, w}}$. In the second case, we set $r_{i_{v, w}}=0$. In all other cases, we set $r_{i_{v, w}}$ to $a_{i_{v, w}}$. Let $\lambda_{r}$ be the partition with the number $r_{i_{v, w}}$ of parts of size $i_{v}$ and color $w$. Let $\lambda_{q}$ be the partition with the number $q_{i_{v, w}}=a_{i_{v, w}}-r_{i_{v, w}}$ of parts of size $i_{v}$ and color $w$. Then we define $G$ be the set of all $\lambda_{r}$ partitions. As $G$ is a finite set, $G(q)$ is the polynomial generating function for the partitions in $G$. Furthermore, by uniqueness of non-negative integer division, we the unique sum $\lambda_{q}+\lambda_{r}=\lambda$.

We verify that $\lambda_{q} \in H$. For the number $q_{i_{v, w}}$ of parts of size $i_{v}$ and color $w$, if there is a $\lambda_{i_{t, u}}^{(A)} \in H$ such that $\lambda_{i_{t, u}}^{(A)}=y \cdot \lambda_{i_{v, w}}^{(A)} \vdash s \cdot i_{t, u}$, then we have two cases. If $s \mid y$, then $y \mid q_{i_{v, w}}$ by definition and hence $q_{i_{v, w}}=k_{i_{t, u}} \cdot y$ for some $0 \leqslant k_{i_{t, u}}<\frac{\operatorname{lcm}(B)}{i_{t, u}}$. If $s / y$, then $\operatorname{gcd}\left(y, \frac{\operatorname{lcm}(B)}{i_{t, u}}\right)=1$ and there is some $0 \leqslant k_{i_{t, u}}<\frac{\operatorname{lcm}(B)}{i_{t, u}}$ such that $y \cdot k_{i_{t, u}} \equiv q_{i_{v, w}}$ $\left(\bmod \frac{\operatorname{lcm}(A)}{i_{v, w}}\right)$. In all other cases, $q_{i_{v, w}}=0$ and hence implicitly is in $H$. Since $\lambda_{q}+\lambda_{r}=\lambda$ is unique, we have satisfied the conditions of Proposition 15, proving the lemma.

Remark 21. The reader is encouraged to show that $H$ is isomorphic to $E_{B}$ (hence a subgroup of $\left.E_{A}\right)$ and that $E_{A} / H \cong G \cong \operatorname{ker}(\varphi)$. This fact is not necessary in the proof but gives an idea how to describe $G$. We can think of the set $G$ as the "remainder" of $E_{A}$ when considered modulo partitions from $E_{B}$.

When $A=\{2, \ldots, m\}$ and $B=\{1, \ldots, m-1\}$, we construct an explicit mapping in the second proof of Lemma 13 between $E_{m \backslash 1}$ and $E_{m-1}$. This mapping inspires the more general proof. Since both $E_{m \backslash 1}$ and $E_{m-1}$ are isomorphic to direct products of cyclic groups, we simply need to describe a homomorphism between these groups. We now sketch the proof Lemma 13 using Lemma 20. This exhibits how Lemma 20 can be used to establish infinite families for partition identities.

Proof. Let $m=s^{k}$ where $s$ is prime and $k$ is a positive integer. By Proposition 18, it is sufficient to define a homomorphism $\varphi: \prod_{i=2}^{m} \frac{\mathbb{Z}_{1 \mathrm{~cm}(m)}}{i} \rightarrow \prod_{i=1}^{m-1} \frac{\mathbb{Z}_{1 \mathrm{~cm}(m-1)}}{i}$. Let $\left(x_{2}, x_{3}, \ldots, x_{m}\right)=$ 
$x \in \prod_{i=2}^{m} \mathbb{Z}_{\frac{\operatorname{lcm}(m)}{i}}$ and $\left(y_{1}, y_{2}, \ldots, y_{m-1}\right)=y \in \prod_{i=1}^{m-1} \mathbb{Z}_{\frac{\operatorname{lcm}(m-1)}{i}}$. The generators of $\prod_{i=2}^{m} \frac{\mathbb{Z}}{\frac{\operatorname{lcm}(m)}{i}}$ are $e_{2}=(1,0,0, \ldots, 0), e_{3}=(0,1,0, \ldots, 0), \ldots, e_{m}=(0,0,0, \ldots, 1)$ and the generators of $\prod_{i=1}^{m-1} \mathbb{Z}_{\frac{\mathrm{lcm}(m-1)}{i}}$ are $f_{1}=(1,0,0, \ldots, 0), f_{2}=(0,1,0, \ldots, 0), \ldots, f_{m-1}=(0,0,0, \ldots, 1)$. We define $\varphi$ based on generators. If $j=s^{r}$ for any $r=1, \ldots, k$, let $\varphi\left(e_{j}\right)=f_{s^{r-1}}$ and $\varphi\left(e_{j}\right)=f_{j}$ otherwise. With $\varphi$ defined, the completion of the proof is routine using $\operatorname{lcm}(m)=s \cdot \operatorname{lcm}(m-1)$ and Lemma 20 and it is left to the reader.

With Lemma 20, we can now generalize Theorem 3.

Theorem 22. Let $A$ and $B$ be two sets of positive integers such that $|A|=|B|$. If there is an onto homomorphism from $E_{A}$ to $E_{B}$, then any constituent of $p(n, A)$ can be expressed as a non-negative linear combination of constituents $p(n, B)$.

Proof. First, by Lemma 20, $E_{A}(q)=G(q) E_{B}\left(q^{s}\right)$ for $s=\frac{l c m(A)}{l c m(B)}$. That is, $G(q)=\frac{E_{A}(q)}{B_{E} q^{s}}$ and can be computed because $E_{A}(q)$ and $E_{B}\left(q^{s}\right)$ are known. We proceed to manipulate the generating function of $p(n, A)$ using the facts noted above,

$$
\begin{aligned}
\sum_{n=0}^{\infty} p(n, A) q^{n} & =\prod_{i \in A} \frac{1}{\left(1-q^{i}\right)}=\frac{E_{A}(q)}{\left(1-q^{l c m(A)}\right)^{d}}=E_{A}(q) \sum_{k=0}^{\infty}\left(\begin{array}{c}
k+d-1 \\
d-1
\end{array}\right) q^{l c m(A) k} \\
& =G(q) E_{B}\left(q^{s}\right) \sum_{k=0}^{\infty}\left(\begin{array}{c}
k+d-1 \\
d-1
\end{array}\right) q^{l c m(A) k}
\end{aligned}
$$

Next, we construct the quasipolynomial for $p(n, B)$,

$$
\sum_{n=0}^{\infty} p(n, B) q^{n}=\prod_{i \in B} \frac{1}{\left(1-q^{i}\right)}=\frac{E_{B}(q)}{\left(1-q^{l c m(B)}\right)^{d}}=E_{B}(q) \sum_{k=0}^{\infty}\left(\begin{array}{c}
k+d-1 \\
d-1
\end{array}\right) q^{l c m(B) k} .
$$

The remainder of the proof follows the proof of Theorem 3 by using the same diligent arithmetic. In particular considering $j(\bmod s)$, that is $j=l^{\prime} s+r$, we have,

$$
p(\operatorname{lcm}(A) k+j, A)=\sum_{i \geqslant 0} c_{r+s i} p\left(\operatorname{lcm}(B) k+l^{\prime}-i, B\right)
$$

The third proof of Lemma 13 using Lemma 20 in conjunction with Theorem 22 proves Theorem 3.

\section{Conclusion}

We have given three proofs of Theorem 3. First, though classic generating function arithmetic. Second, by showing a combinatorial map between 1-free partitions into at most $m$ parts and partitions with no more than $m-1$ parts via conjugation and the sum operation in Section 2. Third, by proving the more general theorem, Theorem 22, which 
applies algebraic structure to $\operatorname{lcm}(A)$-remainder partitions and the sum operation to find a 'universal' mapping. Unifying these proofs is the fact that $p(n, A)$ can be expressed as a quasipolynomial with a finite number of constituents.

The existence of an onto homomoprhism from $E_{A}$ to $E_{B}$ gives a sufficient condition for when a constituent of the quasipolynomial of $p(n, A)$ can be expressed as a non-negative linear combination of constituents of the quasipolynomial of $p\left(n_{1}, B\right)$ with $n_{1} \leqslant n$. The converse is in general not true. For example, Proposition 1 implies that any a constituent of $p(n, m-1)$ can be written as a non-negative linear combination of constituents of $\Delta(n, m)$ by line $(55)$. When $m=5$, there is no onto homomorphism from $E_{4} \rightarrow E_{5 \backslash 1}$ as $\left|E_{5 \backslash 1}\right|>\left|E_{4}\right|$. Given Theorem 3, we pose the following questions.

Question 23. If $m$ is not a prime power, can any constituent of $\Delta(n, m)$ be expressed as a non-negative linear combination of constituents of $p(n, m-1)$ in accordance to Theorem 5 ?

Question 24. What other infinite families of partition identities can be established using Theorem 22?

\section{Acknowledgements}

The author would like to thank Dr. Brandt Kronholm, Dr. Jacob White, and Dr. Timothy Huber for their comments and advice.

\section{References}

[1] Marilena Barnabei, Flavo Bonetti, and Matteo Silimbani, Bijections and Recurrences for Integer Partitions into a Bounded Number of Parts, Appl. Math. Lett. 22 (2009), 297-303.

[2] George Andrews, Matthias Beck, and Neville Robbins, Partitions with fixed differences between largest and smallest parts, Proc. Amer. Math. Soc. 143 (2014).

[3] George E. Andrews, Differences of Partition Functions: The Anti-telescoping Method, From Fourier Analysis and Number Theory to Radon Transforms and Geometry (Hershel M. Farkas, Robert C. Gunning, Marvin I. Knopp, and B. A. Taylor, eds.), Developments in Mathematics, vol. 28, Springer New York, 2013, pp. 1-20 (English).

[4] Felix Breuer, Dennis Eichhorn, and Brandt Kronholm, Polyhedral geometry, supercranks, and combinatorial witnesses of congruences for partitions into three parts, European J. Combin. 65 (2017), 230-252. MR 3679846

[5] Felix Breuer and Brandt Kronholm, A polyhedral model of partitions with bounded differences and a bijective proof of a theorem of Andrews, Beck, and Robbins, Res. Number Theory 2 (2016), Art. 2, 15. MR 3501015

[6] Angelica Castillo, Stephanie Flores, Anabel Hernandez, Brandt Kronholm, Acadia Larsen, and Arturo Martinez, Quasipolynomials and maximal coefficients of gaussian polynomials, Ann. Comb. 23 (2019), 589-611. 
[7] Yuriy Choliy and Andrew V. Sills, A formula for the partition function that "counts", Ann. Comb. 20 (2016), no. 2, 301-316.

[8] Eugène Ehrhart, Sur les polyèdres rationnels homothétiques à n dimensions, C. R. Acad. Sci. Paris 254 (1962), 616-618. MR 0130860 (24 \#A714)

[9] Hansraj Gupta, A technique in partitions, Univ. Beograd. Publ. Elektrotehn Fak ser. Mat. Fiz. (1975), no. 511, 73-76.

[10] Heekyoung Hahn, On classical groups detected by the triple tensor product and the littlewood-richardson semigroup, Res. Number Theory 2 (2016), no. 1, 19.

[11] Heekyoung Hahn, On tensor third L-functions of automorphic representations of $\mathrm{GL}_{n}\left(\mathbb{A}_{F}\right)$, Proc. Amer. Math. Soc. 144 (2016), no. 12, 5061-5069. MR 3556252

[12] Heekyoung Hahn, JiSun Huh, EunSung Lim, and Jaebum Sohn, From partition identities to a combinatorial approach to explicit satake inversion, Ann. Comb. 22 (2018), no. $3,543-562$.

[13] Brandt Kronholm, Ph.d. dissertation, Ph.D. thesis, University at Albany, 1400 Washington Ave, Albany, NY 12222, 102009.

[14] Augustine O. Munagi, Computation of q-partial fractions, Integers 7 (2007), \#A25.

[15] Øystein J. Rødseth and James A. Sellers, Partitions with parts in a finite set, Int. J. Number Theory 2 (2006), no. 3, 455-468.

[16] Andrew V. Sills and Doron Zeilberger, Formule for the number of partitions of $n$ into at most $m$ parts (using the quasi-polynomial ansatz), Adv. in Appl. Math. 48 (2012), no. 5, 640-645.

[17] Richard P. Stanley, Enumerative combinatorics. Volume 1, second ed., Cambridge Studies in Advanced Mathematics, vol. 49, Cambridge University Press, Cambridge, 2012. MR 2868112

[18] E. M. Wright, A simple proof of a known result in partitions, Amer. Math. Monthly 68 (1961), 144-145. 